\title{
Experiences with treprostinil in the treatment of pulmonary arterial hypertension
}

\author{
Amanda R. Stream, MD and \\ University of Colorado Health Sciences Center, Aurora, CO, USA \\ Todd M. Bull, MD \\ University of Colorado Health Sciences Center, Anschutz Medical Campus, Research Building 2, \\ 9th Floor, 12700 East 19th Avenue, Aurora, CO 80045, USA \\ Todd M. Bull: todd.bull@ucdenver.edu
}

\section{Abstract}

Pulmonary arterial hypertension (PAH) is a chronic condition of elevated pulmonary arterial pressures with associated increases in pulmonary vascular resistance leading to right ventricular failure, which was almost uniformly fatal prior to the introduction of pulmonary hypertension specific therapy. Systemic prostacyclin analogs are the first PAH-specific therapies to be made available and are typically recommended as first-line therapy for subjects with severe disease. Treprostinil is a newer prostacyclin analog similar to epoprostenol in its mechanism of action and relative efficacy with the advantage of a longer half life in human serum and room temperature stability. It is unique in that it is available in multiple formulations for alternative routes of delivery, including subcutaneous, intravenous and inhalational routes. Additionally, oral treprostinil is currently under investigation. Both subcutaneous and intravenous forms of treprostinil have demonstrated efficacy in short-term clinical trials and are currently approved for use in subjects with PAH and New York Heart Association functional class (NYHA FC) II-IV symptoms in the USA and for subjects with NYHA FC III and IV in Europe. Inhaled treprostinil has also demonstrated efficacy in short-term clinical trials primarily as add-on therapy and is currently approved for use in subjects with PAH and NYHA FC III-IV symptoms in the USA and Europe. The different formulations of treprostinil have significantly increased the treatment options and opportunities for treatment of patients with PAH.

\section{Keywords}

prostacyclin therapy; pulmonary arterial hypertension; treprostinil

(C) The Author(s), 2012

Correspondence to: Todd M. Bull, todd. bull@ucdenver. edu.

Reprints and permissions: http://www.sagepub.co.uk/journalsPermissions.nav

Conflict of interest statement: The authors disclosed that Todd M. Bull has served on the Advisory board for Actelion. 


\section{Introduction}

Pulmonary hypertension (PH) is a clinical syndrome of inappropriately increased pulmonary arterial pressure (PAP), which can ultimately lead to right ventricular failure and death. The most recent criteria for a diagnosis of PH agreed upon at the 4th World Symposium in Dana Point, California in 2008 is a resting mean PAP of at least $25 \mathrm{mmHg}$ [Simonneau et al. 2009]. Pulmonary arterial hypertension (PAH) is a subset of PH classified as group I under the Dana Point revised World Health Organization (WHO) classification system developed to categorize subsets of $\mathrm{PH}$ by pathogenic mechanisms of disease development. Treatment of PAH includes PAH-specific therapies in addition to conventional therapies such as dietary modifications, supplemental oxygen, diuretics, and for select patients, anticoagulation or digoxin. All current PAH-specific therapy has been developed for and is based upon the current understanding of PAH (WHO group I) pathogenesis.

The pathogenic mechanisms known to contribute to PAH include pulmonary arterial vasoconstriction, platelet activation, in situ thrombosis and vascular remodeling which lead to pre-capillary obstruction and increased pulmonary vascular resistance (PVR). Dysfunction of the pulmonary vascular endothelium is thought to play a key role in the pathogenesis of disease. Prior studies have demonstrated an imbalance in metabolites of arachadonic acid metabolism in pulmonary endothelial cells leading to decreased prostacyclin $\mathrm{I}_{2}$, a potent vasodilator, and increased thromboxane $\mathrm{A}_{2}$, a potent vasoconstrictor, as well as decreased prostacyclin synthase in the lungs of subjects with PAH [Christman et al. 1992; Tuder et al. 1999]. These observations led to the development of prostacyclin analogs currently used in the treatment of PAH.

\section{Prostacyclin therapy for pulmonary arterial hypertension}

Prostacylcin $\mathrm{I}_{2}$ belongs to the family of prostanoids derived from the common precursor prostacyclin $\mathrm{H}_{2}$. It is a short-acting, potent vasodilator produced by the vascular endothelium that also inhibits platelet activation. The prostanoids currently used for treatment of PAH are all synthetic analogs of prostacyclin $\mathrm{I}_{2}$ and include epoprostenol, treprostinil, iloprost and beraprost. Epoprostenol (Flolan, GW USA Inc., Research Triangle, NC, USA) was the first PAH-specific therapy to be approved by the US Food and Drug Administration (FDA) in 1996 and it is the only drug that has demonstrated improved survival in a prospective, randomized, controlled clinical trial specifically for subjects with idiopathic PAH (IPAH) [Barst et al. 1996]. In this pivotal trial evaluating the use of epoprostenol in subjects with IPAH compared with conventional therapy alone there were eight deaths in the conventional therapy group and none in the epoprostenol group over the 12-week study period $(p=0.003)$.

Epoprostenol, however, is unquestionably a difficult medication to administer. It is unstable at room temperature and at $\mathrm{pH}$ levels lower than 10.4, and thus has an estimated half life of only 4-6 min in human plasma. Storage of epoprostenol must be temperature controlled and administration via continuous intravenous infusion. Treatment therefore requires placement of a long-term central venous catheter with the associated risks of infection and thrombosis. Furthermore, due to the short half life and drug instability, brief interruptions in therapy may 
result in rapid deterioration with rebound pulmonary hypertension that is potentially fatal in subjects with severe disease. These characteristics have led to the development of newer prostacyclin derivatives with improved stability and alternative delivery methods.

\section{Treprostinil}

Treprostinil is a tricyclic benzidine prostacyclin analog with similar vasodilatory and antiplatelet effects as epoprostenol [McNulty et al. 1993]. It is the only prostacyclin analog available in multiple formulations with different methods of delivery, including subcutaneous, intravenous, inhalational and oral routes. Unlike epoprostenol, treprostinil is stable in neutral $\mathrm{pH}$ and at room temperature. It has a longer half life, estimated at $2-4 \mathrm{~h}$ in human plasma, and is $100 \%$ bioavailable with rapid absorption following subcutaneous administration [Wade et al. 2004]. The subcutaneous and intravenous forms (Remodulin, United Therapeutics, Silver Spring, MD, USA) are currently recommended for the treatment of subjects with WHO group I PAH and New York Heart Association functional class (NYHA FC) II-IV symptoms in the USA and for subjects with NYHA FC III and IV symptoms in Europe [Badesch et al. 2007; Galie et al. 2009]. The inhalational form of treprostinil (Tyvaso, United Therapeutics) is recommended for use in subjects with NYHA FC III and IV symptoms in both the USA and Europe [Badesch et al. 2007; Galie et al. 2009]. The oral formulation is currently being investigated and is not yet available for use in the USA or elsewhere.

\section{Experience with subcutaneous treprostinil}

The efficacy and safety of treprostinil were first studied in three multicenter pilot clinical trials and the combined results were published by McLaughlin and colleagues [McLaughlin et al. 2003]. Two of these studies were open-label trials, the first compared the acute hemodynamic effects of intravenous epoprostenol with intravenous treprostinil and the second compared a single dose of intravenous treprostinil with three different doses of subcutaneous treprostinil. The results from these trials demonstrated similar hemodynamic improvements with both intravenous and subcutaneous treprostinil compared with epoprostenol with similar dose-limiting toxicities. The third trial was an 8-week placebocontrolled, randomized clinical trial evaluating the longer-term effects of subcutaneous treprostinil on hemodynamics and exercise tolerance compared with placebo. While the results from this pilot study of only 26 subjects did not reach clinical significance, there was a trend towards improved hemodynamic measurements as well as increased exercise tolerance in the treprostinil group, which led to the first ever double-blind, placebocontrolled, randomized clinical trial of PAH-specific therapy.

The multicenter clinical trial published by Simonneau and colleagues included 470 subjects with WHO group I PAH, excluding those with porto-pulmonary hypertension and those with human immunodeficiency virus (HIV)-associated disease [Simonneau et al. 2002]. A total of 469 subjects with NYHA FC II-IV symptoms receiving conventional therapy only prior to enrollment were included in the final analysis. There was a significant difference in the primary efficacy end-point of change in 6 min walk distance (6MWD) from baseline to week 12 for subjects receiving subcutaneous treprostinil therapy compared with those 
randomized to placebo, $10 \mathrm{~m}$ versus $0 \mathrm{~m}$ respectively, with a between-group difference of 16 $\mathrm{m}$ [95\% confidence interval (CI) 4.4-27.6 m, $p=0.006]$. While the overall observed improvement was modest, the improvement in exercise tolerance was greatest in subjects with the most severe limitations at baseline (baseline 6MWD $<150 \mathrm{~m}$ ) and in subjects who achieved the highest doses of treprostinil at week 12. The improvements in exercise tolerance among subjects with more severe disease were similar to those seen with intravenous epoprostenol [Barst et al. 1996] and the authors speculate that the observed effects may have been underestimated due to an inability to reach adequate treprostinil dosing over 12 weeks in some subjects. The most common adverse effects included infusion site pain/reaction and resulted in discontinuation in $18(8 \%)$ subjects receiving the study drug. Other common side effects included those expected with prostacyclin therapy, including headache, diarrhea, nausea and jaw pain. There were no reports of infusion site infections. Serious adverse events included three episodes of gastrointestinal bleeding in the treprostinil group, two of which were associated with excessive anticoagulation and all resolved without adverse consequences.

Barst and colleagues later looked retrospectively at long-term outcomes with subcutaneous treprostinil therapy in an open-label extension study that included subjects who participated in the studies reported by McLaughlin and Simonneau in addition to de novo subjects. Among the de novo subjects, they included subjects with porto-pulmonary hypertension and HIV-associated PAH [Barst et al. 2006]. A total of 860 subjects were enrolled with observation periods of up to 4 years. Reported survival rates from this study for the entire cohort were $87 \%, 78 \%, 71 \%$ and $68 \%$ at years 1, 2, 3 and 4 respectively. For subjects with IPAH for whom baseline hemodynamic data were available, observed survival was improved over that predicted by the National Institute of Health (NIH) equation for IPAH at $91 \%, 82 \%, 76 \%$ and $72 \%$ versus $69 \%, 56 \%, 46 \%$ and $38 \%$ at years $1,2,3$ and 4 , respectively. Conclusions drawn from this study are limited due to a high rate of study drug discontinuation (59\%) and a protocol that allowed for the addition of other PAH-specific therapies at the discretion of treating physicians. A total of 199 (23\%) subjects discontinued therapy due to adverse events, with most (196) reporting injection site pain as the reason for discontinuation; the majority (70\%) occurred within the first year of therapy. There was one death attributed to treprostinil therapy in a subject with progressive PAH. The authors conducted survival analyses using only subjects receiving subcutaneous treprostinil monotherapy and only those who received subcutaneous treprostinil for at least 1 year. There were no significant differences in corrected survival rates compared with the observed crude rates for the entire study cohort.

A second long-term, open-label trial of subcutaneous treprostinil efficacy in 122 subjects with PAH, including 23 subjects with chronic thromboembolic pulmonary hypertension (CTEPH; WHO group IV PH), was reported by Lang and colleagues [Lang et al. 2006]. The findings from this study were similar to those reported by Barst and colleagues, with overall survival rates at 1 and 3 years of $88.6 \%$ and $70.6 \%$, respectively, and no subjects received additional PAH-specific therapy. In contrast to the study by Barst and colleagues, the reported discontinuation rate of the study drug by Lang and colleagues was $10.6 \%$, with only six subjects (4.9\%) reporting injection site pain as the reason for discontinuation 
despite its occurrence in $82 \%$ of study subjects. Lastly, a more recent retrospective analysis by Sadushi-Kolici and colleagues evaluated outcomes over a 10-year time period among 111 subjects with severe (NYHA FC III and IV) PH, 62\% of whom were WHO group I [Sadushi-Kolici et al. 2012]. Interestingly, 38\% of subjects included were diagnosed with CTEPH (WHO group IV) which was inoperable, a group rarely included in treatment trials. They demonstrated good tolerability with a $12 \%$ discontinuation rate for drug-related side effects. Reported survival rates were $84 \%, 53 \%$ and $33 \%$ at 1,5 and 9 years respectively with a promising survival rate of $57 \%$ at 9 years for subjects able to tolerate treatment for greater than 9 months. There were no significant differences in survival between subjects with WHO group I PAH and those with CTEPH (WHO group IV). The results from these studies suggest that subcutaneous treprostinil is a safe and efficacious alternative to intravenous epoprostenol for subjects with WHO group I PAH and NYHA FC II-IV symptoms who are poor candidates for or wish to avoid the risks associated with chronic indwelling central venous catheters.

\section{Experience with intravenous treprostinil}

Injection site pain is common across clinical trials of subcutaneous treprostinil with varying tolerability, as demonstrated by the differing rates of study drug discontinuation attributed to this adverse effect among trial participants. While subcutaneous administration is one of the major perceived benefits of treprostinil over epoprostenol by avoiding the risks of catheterrelated infections and thrombosis, the stability and longer half life of intravenous treprostinil may still render it preferable to epoprostenol in subjects unable to tolerate treprostinil via the subcutaneous route. To this end, Tapson and colleagues studied the safety and efficacy of intravenous treprostinil in a prospective, multicenter, open-label clinical trial [Tapson et al. 2006]. This 12-week study included 16 subjects with WHO group I PAH naïve to other PAH-specific therapy. Of the 14 subjects completing the study protocol (13 with NYHA FC III and 1 with FC IV symptoms), eight had stabilization of their NYHA FC symptoms and six improved in FC. There was a significant increase in mean 6MWD both at week $6(59 \pm$ $19 \mathrm{~m}, p=0.008)$ and at week $12(82 \mathrm{~m}$, from baseline $319 \pm 22 \mathrm{~m}$ to $400 \pm 26 \mathrm{~m}, p=0.001)$. Observed hemodynamic improvements included a significant decrease in mean PAP ( $-9 \%$, $p=0.03), \operatorname{PVR}(-33 \%, p=0.001)$ and a significant increase in cardiac index $(+29 \%, p=$ 0.002 ) from baseline to week 12. There were no serious adverse events reported.

Given the relative efficacy and perceived benefits of intravenous treprostinil over intravenous epoprostenol, Gomberg-Maitland and colleagues evaluated the safety and efficacy of transition from intravenous epoprostenol to intravenous treprostinil [GombergMaitland et al. 2005]. This 12-week open-label, investigator-initiated multicenter trial included 31 subjects with WHO group I PAH and NYHA FC II or III symptoms who had been receiving a stable dose of continuous intravenous epoprostenol for a period of at least 3 months prior to study enrollment. Subjects were hospitalized for the initial transition and uptitration of intravenous treprostinil with further dose increases occurring over the 12-week observation period. The primary endpoint was change in 6MWD assessed at baseline on epoprostenol and at week 12 following transition to intravenous treprostinil. Secondary endpoints included changes in hemodynamic parameters assessed by right heart catheterization (RHC), Borg dyspnea score, NYHA FC symptoms, and exercise tolerance 
assessed by the Naughten-Balke treadmill test. Among the 37 subjects who completed the study protocol, there was no significant difference in the primary endpoint of 6MWD or in any of the secondary endpoints from baseline on epoprostenol to week 12 on intravenous treprostinil. There were no deaths during the study observation period and the most common adverse effect was extremity pain (71\%) which led to transition back to epoprostenol in three subjects. Only one subject was transitioned back to epoprostenol for worsening PAH and this was in the setting of pneumonia. An important observation from this study was that the relative potency of intravenous treprostinil appears to be roughly half that of equivalent epoprostenol dosing. The authors speculate that the higher required doses of treprostinil may have contributed to the apparent increased incidence of prostacyclin-related side effects in some subjects.

Data comparing intravenous treprostinil with placebo have recently been reported by Hiremath and colleagues [Hiremath et al. 2010]. This trial, performed in India where there are no routinely available PAH-specific therapies, was a short-term, placebo-controlled trial of intravenous treprostinil among subjects with WHO group I PAH and FC III or IV symptoms naïve to PAH specific therapy. The study was originally planned to be of similar size and duration to the initial study of intravenous epoprostenol in PAH reported by Barst and colleagues [Barst et al. 1996]; however this randomized, double-blind, placebocontrolled trial ended early due to an exceptionally high rate of serious adverse events among both the placebo (64\%) and study drug (37\%) participants, primarily related to catheter complications and infection. Enrollment was ended after 45 out of a planned 126 subjects were randomized and 44 subjects who received treatment were included in the final safety and efficacy analyses. In total 31 subjects completed the 12-week protocol. There was a significant increase in 6MWD from baseline to week 12 favoring the intravenous treprostinil-treated subjects with a median between-group difference of $83 \mathrm{~m}$ (95\% CI 7-187 $\mathrm{m}, p=0.0077)$. There was also a trend towards improved survival favoring the treprostiniltreated group ( $p=0.051)$. While the results are informative and promising, this study highlights the risks associated with catheter use and placement and the importance of center experience in the administration of intravenous prostanoid therapy.

Intravenous treprostinil appears to be both safe and efficacious for subjects with WHO group I PAH and NYHA FC II-IV symptoms. Continuous intravenous treprostinil, like subcutaneous treprostinil, is approved for use in subjects with WHO group I PAH and NYHA FC II-IV symptoms in the USA and for subjects with NYHA FC III and IV symptoms in Europe.

There are no studies published to date comparing intravenous epoprostenol and intravenous treprostinil nor are there studies of long-term outcomes with intravenous treprostinil. In addition, there are no data comparing first-line parenteral prostacyclin therapy with upfront oral combination therapy, which is of particular interest given the apparent complementary effects of combination therapy observed in trials of add-on combination therapy. Furthermore, the timing of initiation with regards to NYHA FC symptoms has not been explicitly addressed in clinical trials. Reflective of this lack of evidence, European and American expert consensus guidelines differ in regards to recommendations for parenteral therapy use in subjects with NYHA FC II symptoms; however, parenteral or oral therapy is 
recommended for consideration by both for subjects with NYHA FC III symptoms. Based on current data and until further evidence is available we recommend parenteral prostacyclin therapy for subjects with NYHA FC III or IV symptoms and other high-risk clinical features, as described by McLaughin and McGoon, if the patient is capable of safely selfadministering the medication [McLaughlin and McGoon, 2006].

\section{Experience with inhaled treprostinil}

The same year that intravenous treprostinil was FDA approved for treatment of PAH, iloprost, the third prostacyclin analog and first inhaled therapy for PAH, was also approved for use in subjects with WHO group I PAH and NYHA FC III or IV symptoms. In a placebo-controlled clinical trial of efficacy, inhaled iloprost (Ventavis, Actelion, London, UK) demonstrated a significant improvement in the composite endpoint of improved exercise tolerance, improved NYHA FC and decreased deterioration in subjects with NYHA FC III or IV symptoms compared with those receiving placebo [Olschewski et al. 2002]. While the inhaled route is less invasive than intravenous or subcutaneous infusion and therefore may be the preferred route of administration for patient safety and comfort, the dosing regimen for iloprost is cumbersome. Owing to its short half life (20-30 min in human serum) and rapid elimination time (30 min to $1 \mathrm{~h}$ ), iloprost must be administered via six to nine inhalations as frequently as every $2 \mathrm{~h}$ while awake.

As stated previously, treprostinil has a longer half life of $2-4 \mathrm{~h}$ in human serum as well as alternative tissue binding, making it an attractive candidate for inhalational therapy. Voswinckel and colleagues evaluated the acute vasodilatory effects of inhaled treprostinil by RHC compared with inhaled iloprost and explored the maximum tolerated doses as well as minimum inhalation time for efficient dosing in a series of three open-label observational studies [Voswinckel et al. 2006]. They observed equivalent acute vasodilatory effects between the two drugs, with slightly increased time to effect but longer duration of action with treprostinil compared with iloprost. In addition, treprostinil was well tolerated with less systemic hypotension than seen with iloprost and a duration of effect that appeared to last beyond the time of detectable serum concentrations, suggesting increased pulmonary selectivity with treprostinil.

The longer-term clinical efficacy of inhaled treprostinil was evaluated in an open-label 12week pilot study as add-on therapy in subjects receiving the oral endothelin receptor antagonist bosentan [Channick et al. 2006]. This study enrolled 12 subjects with NYHA FC III symptoms despite treatment with bosentan for a minimum of 12 weeks. Participants received either 30 or $45 \mu \mathrm{g}$ of inhaled treprostinil delivered via six to nine inhalations, four times daily. Among the 11 subjects who completed the study there was a trend towards improvement in 6MWD of more than $10 \%$ from baseline for subjects receiving $45 \mu \mathrm{g}$ inhaled treprostinil and 9 out of all 11 subjects reported improved NYHA FC symptoms from III to II. All subjects tolerated the therapy well with no serious adverse events. The most common adverse effect reported was transient cough.

From these initial observations came the TRIUMPH-1 (Treprostinil Inhalation Used in the Management of Pulmonary Arterial Hypertension) study [McLaughlin et al. 2010]. This was 
a 12-week multicenter, double blind, placebo-controlled, randomized clinical trial evaluating the safety and efficacy of inhaled treprostinil as add-on therapy to either bosentan or the oral phosphodiesterase-5 inhibitor sildenafil. A total of 235 subjects with WHO group I PAH and NYHA FC III or IV symptoms were enrolled, 115 randomized to treprostinil and 110 to placebo. Twenty-three subjects withdrew prematurely (13 study subjects and 10 placebo subjects), although all subjects were included in both efficacy and safety analyses. The primary efficacy endpoint was change in 6MWD from baseline to week 12 while secondary analyses included time to clinical worsening, Borg dyspnea score, NYHA FC symptoms, quality of life assessments and change in 6MWD at week 6. The median between-group difference in change in 6MWD from baseline to week 12 was $+20 \mathrm{~m}(95 \%$ CI 8-32.8, $p=$ 0.004 ) favoring inhaled treprostinil over placebo. When stratified by background therapy there remained a significant improvement among subjects treated with bosentan, with a median between-group difference in 6MWD at week 12 of $25 \mathrm{~m} \mathrm{(95 \%} \mathrm{CI} \mathrm{10.2-40,} p=$ 0.0002) while the between-group difference in 6MWD for subjects taking sildenafil was no longer significant. There were no significant differences in secondary endpoints except for quality of life assessed by the Minnesota Living with Heart Failure Questionnaire (MLHFQ), with significant improvements in both the global and physical domains for subjects receiving treprostinil. There were 11 serious adverse events reported among the treprostinil group and only one death, which occurred in the placebo group. The most common adverse effect was cough, reported by $54 \%$ of subjects receiving treprostinil and $29 \%$ of subjects receiving placebo.

In an open-label extension study of TRIUMPH-1, Benza and colleagues followed 206 subjects for a period of 2 years to assess long-term clinical efficacy [Benza et al. 2011]. Participants were assessed at 3-month intervals using 6MWD, Borg dyspnea score, NYHA FC symptoms, quality of life assessment as well as reporting of adverse events. Eighty-four subjects discontinued therapy over the 2-year observation period, 40 reporting adverse effects as the reason for discontinuation, including progression of $\mathrm{PAH}$, cough and headache. There were 14 deaths, none of which were attributed to treprostinil therapy. Cough was the most common adverse effect, occurring in $53 \%$ of subjects. Of the 122 subjects who received treprostinil for 24 months or longer, there was a persistent improvement in 6MWD compared with baseline, stabilization or improvement in NYHA FC and improved Borg dyspnea and quality of life scores over the period of observation. There are no data from controlled trials on the efficacy of inhaled treprostinil as monotherapy, although current data suggest that it is an effective add-on therapy. Inhaled treprostinil presents an additional treatment modality with limited side effects that may be preferable for subjects who are not good candidates for or wish to avoid continuous infusion therapy. Inhaled treprostinil is currently approved for treatment of subjects with WHO group I PAH with NYHA class III or IV symptoms in both the USA and Europe.

\section{Experiences with oral treprostinil}

The oral formulation of treprostinil has recently been studied as both monotherapy and addon therapy in three double-blind, placebo-controlled, randomized clinical trials. The results from the first of these trials, FREEDOM-C, have recently been published [Tapson et al. 2012]. This study evaluated the efficacy and safety of oral treprostinil as add-on therapy 
among 39 subjects on background endothelin receptor antagonist, phosphodiesterase-5 inhibitors or both over a 16-week treatment period. The primary efficacy endpoint of placebo-corrected change in 6MWD from baseline was not met; however, there appeared to be a dose-dependent effect with subjects able to tolerate higher doses of oral treprostinil experiencing larger improvements in 6MWD. The authors speculate that dose escalation may have been limited by side effects and the limited availability of lower-dose tablets for slower uptitration. The results from two additional studies are pending publication and an additional, longer-term study of oral treprostinil as add-on therapy is currently being planned. Oral treprostinil is not yet approved for use.

\section{Future directions}

In addition to the trials of oral treprostinil, other ongoing studies include the safety and efficacy of transition from infusion (either subcutaneous or intravenous) to oral treprostinil, transition from infusion to inhaled treprostinil, transition from inhaled iloprost to inhaled treprostinil and long-term safety and efficacy following transition from intravenous epoprostenol to intravenous treprostinil. A surgically implanted pump for continuous infusion therapy is currently in development in an attempt to improve patient satisfaction and decrease infectious complications associated with parenteral delivery but has not yet been formally studied. Lastly, inhaled treprostinil, with its apparent pulmonary specificity, is being evaluated for use in subjects with $\mathrm{PH}$ associated with hypoxic lung disease (WHO group III).

\section{Conclusions}

Significant advances have been made in the treatment of PAH since the approval of continuous intravenous epoprostenol in 1996. While it remains the only therapy to have demonstrated a survival benefit in a prospective trial for a select group of patients (IPAH), the availability of effective therapies has rendered placebo-controlled trials of newer treatments difficult if not impossible to perform in subjects with severe disease due to the high mortality in this patient population. Treprostinil, due to its pharmacological stability, offers the benefit of PAH therapy in multiple formulations for subjects with a broad range of NYHA FC symptoms (II-IV). The longer serum half life adds convenience and safety for parenteral application and the subcutaneous, inhaled and perhaps oral formulations expand the patient population able to receive a prostanoid analog. However, many areas of uncertainty regarding the use of treprostinil still exist, some of which are being addressed in ongoing clinical trials. These include optimal timing for initiation of infusion therapy, whether inhaled or oral therapy is as efficacious as infusion therapy in the long-term, and when and how transition from one modality to the other should take place. Despite these limitations it is clear that therapeutic advancements in the field of PAH have improved survival overall compared with historical controls and with multiple options available, treatment decisions should be made based on current guidelines, clinical severity, and individual patient preference as well as feasibility. 


\section{Acknowledgments}

Funding: The authors declared that Todd M. Bull has received an Investigator initiated grant from United Therapeutics.

\section{References}

Badesch D, Abman S, Simonneau G, Rubin L, McLaughlin V. Medical therapy for pulmonary arterial hypertension: updated ACCP evidence-based clinical practice guidelines. Chest. 2007; 131:19171928. [PubMed: 17565025]

Barst R, Galie N, Naeije R, Simonneau G, Jeffs R, Arneson C, et al. Long-term outcome in pulmonary arterial hypertension patients treated with subcutaneous treprostinil. Eur Respir J. 2006; 28:11951203. [PubMed: 16899485]

Barst R, Rubin L, Long W, McGoon M, Rich S, Badesch D, et al. A comparison of continuous intravenous epoprostenol (prostacyclin) with conventional therapy for primary pulmonary hypertension. The Primary Pulmonary Hypertension Study Group. N Engl J Med. 1996; 334:296302. [PubMed: 8532025]

Benza R, Seeger W, McLaughlin V, Channick R, Voswinckel R, Tapson V, et al. Long-term effects of inhaled treprostinil in patients with pulmonary arterial hypertension: the Treprostinil Sodium Inhalation Used in the Management of Pulmonary Arterial Hypertension (TRIUMPH) study openlabel extension. J Heart Lung Transplant. 2011; 30:1327-1333. [PubMed: 22055098]

Channick R, Olschewski H, Seeger W, Staub T, Voswinckel R, Rubin L. Safety and efficacy of inhaled treprostinil as add-on therapy to bosentan in pulmonary arterial hypertension. J Am Coll Cardiol. 2006; 48:1433-1437. [PubMed: 17010807]

Christman B, McPherson C, Newman J, King G, Bernard G, Groves B, et al. An imbalance between the excretion of thromboxane and prostacyclin metabolites in pulmonary hypertension. N Engl J Med. 1992; 327:70-75. [PubMed: 1603138]

Galie N, Hoeper M, Humbert M, Torbicki A, Vachiery J, Barbera J, et al. Guidelines for the diagnosis and treatment of pulmonary hypertension. Eur Resp J. 2009; 34:1219-1263.

Gomberg-Maitland M, Tapson V, Benza R, McLaughlin V, Krichman A, Widlitz A, et al. Transition from intravenous epoprostenol to intravenous treprostinil in pulmonary hypertension. Am J Resp Crit Care Med. 2005; 172:1586-1589. [PubMed: 16151039]

Hiremath J, Thanikachalam S, Parikh K, Shanmugasundaram S, Bangera S, Shapiro L, et al. Exercise improvement and plasma biomarker changes with intravenous treprostinil therapy for pulmonary arterial hypertension: a placebo-controlled trial. J Heart Lung Transplant. 2010; 29:137-149. [PubMed: 20022264]

Lang I, Gomez-Sanchez M, Kneussl M, Naeije R, Escribano P, Skoro-Sajer N, et al. Efficacy of longterm subcutaneous treprostinil sodium therapy in pulmonary hypertension. Chest. 2006; 129:16361643. [PubMed: 16778286]

McLaughlin V, Benza R, Rubin L, Channick R, Voswinckel R, Tapson V, et al. Addition of inhaled treprostinil to oral therapy for pulmonary arterial hypertension: a randomized controlled clinical trial. J Am Coll Cardiol. 2010; 55:1915-1922. [PubMed: 20430262]

McLaughlin V, Gaine S, Barst R, Oudiz R, Bourge R, Frost A, et al. Efficacy and safety of treprostinil: an epoprostenol analog for primary pulmonary hypertension. J Cardiovasc Pharmacol. 2003; 41:293-299. [PubMed: 12548091]

McLaughlin V, McGoon M. Pulmonary arterial hypertension. Circulation. 2006; 114:1417-1431. [PubMed: 17000921]

McNulty M, Sailstad J, Steffen R. The pharmacokinetics and pharmacodynamics of the prostacyclin analog 15AU81 in the anesthetized beagle dog. Prostaglandins Leukot Essent Fatty acids. 1993; 48:159-166. [PubMed: 8446654]

Olschewski H, Simonneau G, Galie N, Higenbottam T, Naeije R, Rubin LJ, et al. Inhaled iloprost for severe pulmonary hypertension. N Engl J Med. 2002; 347:322-329. [PubMed: 12151469] 
Sadushi-Kolici R, Skoro-Sajer N, Zimmer D, Bonderman D, Schemper M, Klepetko W, et al. Longterm treatment, tolerability, and survival with sub-cutaneous treprostinil for severe pulmonary hypertension. J Heart Lung Transplant. 2012; 31:735-743. [PubMed: 22480725]

Simonneau G, Barst R, Galie N, Naeije R, Rich S, Bourge R, et al. Continuous subcutaneous infusion of treprostinil, a prostacyclin analogue, in patients with pulmonary arterial hypertension: a doubleblind, randomized, placebo-controlled trial. Am J Resp Crit Care Med. 2002; 165:800-804. [PubMed: 11897647]

Simonneau G, Robbins I, Beghetti M, Channick R, Delcroix M, Denton C, et al. Updated clinical classification of pulmonary hypertension. J Am Coll Cardiol. 2009; 54(1 Suppl):S43-S54. [PubMed: 19555858]

Tapson V, Gomberg-Maitland M, McLaughlin V, Benza R, Widlitz A, Krichman A, et al. Safety and efficacy of IV treprostinil for pulmonary arterial hypertension: a prospective, multicenter, openlabel, 12-week trial. Chest. 2006; 129:683-688. [PubMed: 16537868]

Tapson V, Torres F, Kermeen F, Keogh A, Allen R, Frantz R, et al. Oral treprostinil for the treatment of pulmonary arterial hypertension in patients on background endothelin receptor antagonist and/or phosphodiesterase type 5 inhibitor therapy (the FREEDOM-C study): a randomized controlled trial. Chest. 2012 May 24. (epub ahead of print).

Tuder R, Cool C, Geraci M, Wang J, Abman S, Wright L, et al. Prostacyclin synthase expression is decreased in lungs from patients with severe pulmonary hypertension. Am J Resp Crit Care Med. 1999; 159:1925-1932. [PubMed: 10351941]

Voswinckel R, Enke B, Reichenberger F, Kohstall M, Kreckel A, Krick S, et al. Favorable effects of inhaled treprostinil in severe pulmonary hypertension: results from randomized controlled pilot studies. J Am Coll Cardiol. 2006; 48:1672-1681. [PubMed: 17045906]

Wade M, Baker F, Roscigno R, DellaMaestra W, Hunt T, Lai A. Absolute bioavailability and pharmacokinetics of treprostinil sodium administered by acute subcutaneous infusion. J Clin Pharmacol. 2004; 44:83-88. [PubMed: 14681345] 